\title{
Changes in Platelet Count and Related Parameters in SART- Stressed Mice and the Action of Administered Neurotropin
}

\author{
Taeko HATA, Atsufumi KAWABATA, Tomitaro KITA, \\ Eiji ITOH and Yoshitaka NISHIMURA \\ Department of Pharmacology. Faculty of Pharmacy. Kinki University. \\ Kowakae, Higashi-Osaka 577. Japan \\ Accepted April 21, 1988
}

\begin{abstract}
In order to hematologically characterize SART-stressed (repeated coldstressed) animals, which are regarded as model animals of clinical dysautonomia, general hematological analyses were performed in mice subjected to various types of stress. SART-stressed mice showed significant increases in erythrocyte count. hemoglobin, hematocrit and specific gravity of whole blood, no change in leukocyte count and a marked decrease in platelet count. Among the above changes, the decreased platelet count was particularly characteristic of SART-stressed mice. Splenectomy failed to inhibit the SART stress-induced thrombocytopenia. Bone marrow megakaryocyte counts increased following the stress. The bleeding time of SART-stressed mice was more than double that of normal mice. Consecutive administrations of Neurotropin a sedative analgesic, completely blocked the alterations in platelet count, megakaryocyte count and bleeding time in SARTstressed mice without producing any effect in unstressed mice. From the present results, it is suggested that SART-stressed mice may be characterized by thrombocytopenia. which is not attributable to enhanced function of the spleen or suppressed platelet production in the bone marrow. Moreover. Neurotropin appears to be effective for moderating SART stress.
\end{abstract}

It has been known for a long time that stressful stimuli can cause various hematological changes including eosinopenia. Among the hematological responses to stress, however, some seem to vary with the type, severity and duration of stress employed (1-3).

The SART (specific alternation of rhythm in temperature)-stressed (repeated coldstressed) animal (4,5) has been regarded as a model of clinical dysautonomia (6), showing a variety of physiological anomalies including continuous hypotension (7), altered blood flow (7, 8), electrocardiographic (9) and electroencephalographic (10) abnormalities and hyperalgesia (11). Such animals have also been reported to exhibit certain biochemical changes such as decreased levels of acetylcholine (12) and increased levels of catecholamines (13) in the brain. The majority of the foregoing alterations caused by SART stress differ from responses to common acute stress and are long-lasting and unresponsive to subsequent change.

With respect to their hematological profile. SART-stressed animals have been reported to show increased erythrocyte and neutrophil counts and decreased lymphocyte and eosinophil counts (14), whereas no alterations characteristic of this form of stress have been determined. In the initial part of the present study, therefore, common blood analyses were undertaken in mice subjected to various types of stress in order to characterize SARTstressed animals hematologically and to explore a reference point for making a clinical diagnosis of dysautonomia.

The results indicated that thrombocytopenia might be characteristic of SARTstressed mice, and therefore subsequent experiments were designed in order to evaluate this phenomenon in more detail.

Moreover, the effect of Neurotropin ${ }^{\mathrm{i}}$, a 
sedative analgesic, on SART stress-induced changes in platelet count and related parameters was studied. Neurotropin is an extract isolated from the inflamed skin of rabbits inoculated with vaccinia virus, and it has been reported to be effective in alleviating most of the abnormal symptoms caused by SART stress $(6-9,12,14,15)$. Similarly, the action of alprazolam, a minor tranquilizer, was examined and compared with that of Neuro tropin.

\section{Materials and Methods}

Male ddY strain mice weighing about $25 \mathrm{~g}$ were used throughout these experiments.

\section{Stress procedure}

SART stress: Mice were alternately exposed to room temperatures of $24^{\circ} \mathrm{C}$ and $4^{\circ} \mathrm{C}$ at $1-\mathrm{hr}$ intervals from 09:00 to $16: 00$, and then kept at $4^{\circ} \mathrm{C}$ from $16: 00$ to $09: 00$ the following morning (5). This procedure was repeated for 7 consecutive days, unless otherwise stated. Then, the stressed mice were subjected to experiments one or more hr after the termination of stress on the final morning.

Cold stress: Mice were kept at $4{ }^{\circ} \mathrm{C}$ continuously for 2 days.

Restraint plus water immersion stress (RWIS): Each mouse was restrained in a wire cylinder and immersed vertically in water at $15^{\circ} \mathrm{C}$ up to the xiphoid process for $1 \mathrm{hr}$ ( 8 . 16).

Restraint stress: Mice were restrained in the same manner at room temperature for 15 hr overnight from 19:00 to 10:00 the following morning. As a control, mice were deprived of food and water according to the same schedule.

\section{General hematological analysis}

Under light ether anesthesia, blood samples were obtained from mice by cardiac puncture after thoracotomy. Estimations of red blood cell (RBC) and white blood cell (WBC) counts, hemoglobin $(\mathrm{Hb})$ and hematocrit $(\mathrm{Ht})$ were simultaneously performed automatically by the usual methods. The specific gravity of whole blood $\left(G_{B}\right)$ was determined by the copper sulfate method (17). Platelets were visually counted by the method of Brecher and Cronkite (18) using a phase-contrast microscope, since the platelets of mice are much smaller and greater in number than those of humans. For this estimation, the tails of conscious mice were cut $1 \mathrm{~cm}$ from the tip and $10 \mu l$ of exuded blood was collected directly using a micropipette. All blood analyses were performed between 10:00 and $12: 00$ in consideration of circadian fluctuations (19).

\section{Bone marrow megakaryocyte counts}

Mice were killed by cervical dislocation. both femurs were removed, and the internal bone marrow megakaryocytes were counted as described by Nomura (20). The marrow of each femur was gently flushed out into $1 \mathrm{ml}$ of $5 \%$ EDTA-2Na solution. The dispersed cell suspension was stained by $0.025 \%$ gentian violet, and the megakaryocytes were counted visualiy through a light microscope using a Fuchs-Rosenthal hemacytometer. Megakaryocyte counts were expressed as total cell counts per femur, and the average of the values obtained from both femurs was taken for each mouse.

\section{Bleeding time}

Bleeding time was measured according to the tail transection technique of O'Neil (21). Unanesthetized mice were restrained in an opaque plastic cylinder with the tail exposed. The tail was immersed in isotonic $0.9 \%$ saline solution maintained at $37^{\circ} \mathrm{C}$. After a 5 -min resting period, a $1-\mathrm{cm}$ segment was cut from the tip of the tail with a sharp safety razor, and the tail was immediately placed back into the saline solution. Bleeding time was defined as the time from the moment the cut was made until complete cessation of bleeding without any rebleeding within $30 \mathrm{sec}$.

\section{Splenectomy}

Under pentobarbital (Abott. Nembutal ${ }^{\circledR}$ ) anesthesia, the spleen was exposed via a left side incision. After the major blood vessels had been ligated with sutures, the spleen was excised. As a sham operation, the spleen was exposed in the same manner but not excised. In a preliminary experiment, the increase in platelet count was maximal and steady $7-9$ days after splenectomy. Then, in the subsequent experiment, platelet counting was carried out 8 days after splenectomy, and mice were loaded with SART stress for 6 days from 2 days after the operation.

\section{Drug administration}

The drugs used were Neurotropin (Nippon 
Zoki) and alprazolam (Takeda). Neurotropin. undiluted solution $(20 \mathrm{mg} / \mathrm{ml})$, and alprazolam suspended in $0.5 \%$ CMC- $\mathrm{Na}$ solution were administered i.p. and p.o. to mice. respectively. For the evaluation of acute effects, mice were given the drugs only once $60 \mathrm{~min}$ before the measurements. SART. stressed mice were also treated with a single dose after 7 days of stress loading. and the measurements were carried out 60 min later. In a chronic study. mice received the drugs once daily. 7 times in all. until the day preceding the measurements. Similarly. SARTstressed mice were treated with daily doses during 7 days of stress loading. The measurements were carried out on the day following the final dose.

\section{Statistical analysis}

The results obtained were represented as the mean \pm S.E. Statistical evaluation was performed using Student's t-test for unpaired data or analysis of variance followed by the Student-Newman-Keuls test for multiple comparisons; data were considered statistically significant at $P$ values of less than 0.05 .

\section{Results}

1. Hematological profiles of mice exposed to various types of stress

As shown in Fig. 1, all forms of stress with the exception of simple cold stress caused significant increases in $\mathrm{RBC}$ and $\mathrm{Ht}$. $\mathrm{Hb}$ and $\mathrm{G}_{B}$ were significantly increased by SART stress, RWIS and fasting, but not by cold and restraint stress. No change in WBC occurred with SART and cold stress, al though a striking decrease was observed with RWIS, restraint and fasting. Platelet count was significantly increased by exposure to cold and restraint. In contrast, exposure to SART stress and RWIS resulted in markedly decreased platelet counts.

Thus, decreased platelet counts and unchanged WBC seemed characteristic of SART-stressed mice.

2. Influence of splenectomy on thrombocytopenia in SART-stressed mice

In order to determine whether SART stress-induced thrombocytopenia was associated with altered splenic function, splenectomized mice were subjected to SART
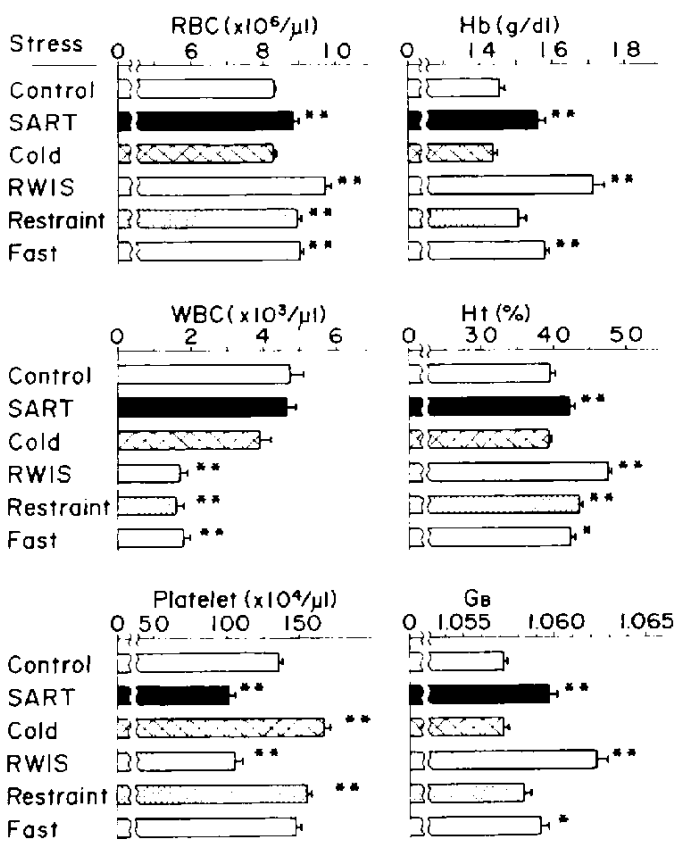

Fig. 1. Hematological profles in stressed mice. $n=7 \cdots 13$ *P $\mathrm{P}<0.05$ ** P 0.01

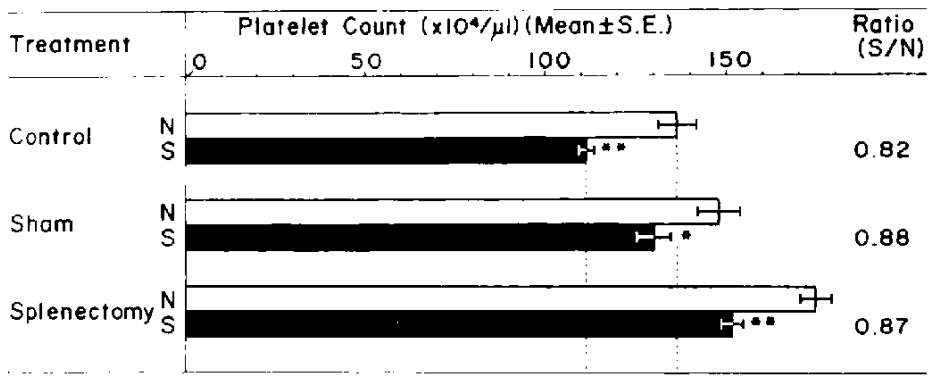

Fig. 2. Influence of splenectomy on decreased platelet count in SART-stressed mice. N. Non-stress: S. SART stress. $\quad n=7-.10 . \quad P<0.05,{ }^{*} \mathrm{P} * 0.01$, compared to the respective non-stressed groups. 
stress.

As seen in Fig. 2, splenectomy caused markedly increased platelet counts in both unstressed and SART-stressed mice ( $P<0.01$ vs. control), although a slight rise was also noted in the sham-operated groups. The ratio of the platelet count for SART-stressed mice to the non-stressed value in the splenectomized group (0.87) was similar to that in the sham-operated group (0.88). In other words, splenectomy failed to inhibit the SART stress-induced fall in platelet count.

3. Relationship between changes in platelet and bone marrow megakaryocyte counts in SART-stressed mice

Platelet counts were $135.2 \pm 3.8$ and 102.1 $\pm 2.3 \times 10^{4} / u 1$ in non-stressed and SARTstressed mice, respectively $(P<0.001)$, and megakaryocyte counts were $7,406 \pm 409$ and
$10.332 \pm 212 /$ femur, respectively $(P<0.001)$. Megakaryocyte counts were strikingly increased by about $40 \%$ following SART stress. while platelet counts were decreased by about $25 \%$.

Figure 3 illustrates the time-related changes in platelet and megakaryocyte counts during SART stress. Platelet count remained within normal limits up to day 3 of stress and then significantly decreased from day 5 , reaching a minimum on day 7 . The decreased cell counts were maintained at least up to day 14 . On the other hand, megakaryocyte counts were significantly increased from day 5 and reached a plateau on day 7 . the increase being maintained during an additional one week of stress. Thus, an inverse correlation existed between changes in platelet and megakaryocyte counts during SART stress.

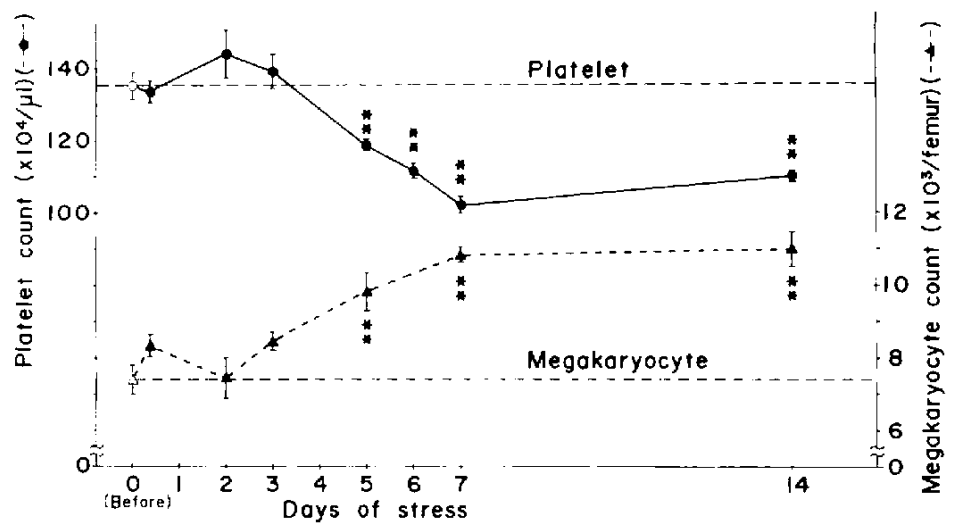

Fig. 3. Time courses of SART stress-induced changes in platelet and megakaryocyte counts in mice. $n=7-10 . \quad * P<0.01$, compared to the initial level.

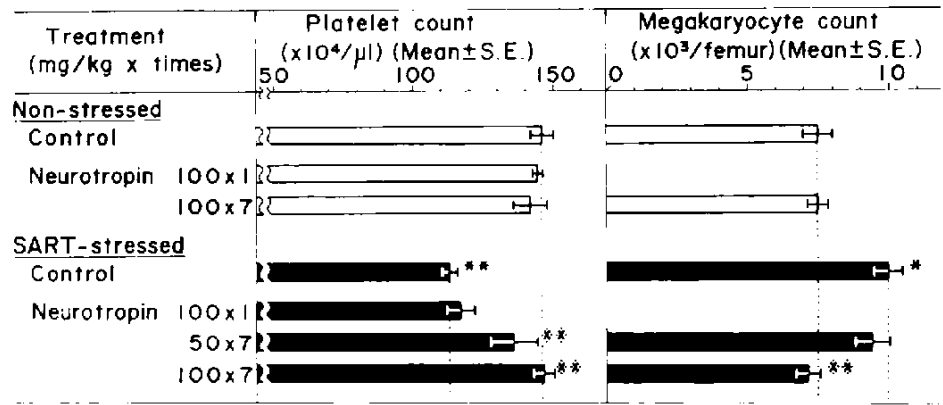

Fig. 4. Effect of Neurotropin on SART stress-induced changes in platelet and megakaryocyte counts in mice. $n=6-12$. ${ }^{*} P<0.05,{ }^{*} P<0.01$. compared to the non-stressed control. * * $P<0.01$, compared to the SART-stressed control. 
4. Changes in bleeding time caused by SART stress

Bleeding time of SART-stressed mice was $130.5 \pm 9.1 \mathrm{sec}$ ( $P<0.001 \mathrm{vs}$. normal level). which was more than double that in normal mice, $55.2 \pm 4.1 \mathrm{sec}$.

Since, as described above, SART-stressed mice showed thrombocytopenia, megakaryocytosis and prolonged bleeding time, the effects of drugs on these alterations were examined in subsequent experiments.

5. Actions of drugs on platelet count and related parameters in unstressed and SARTstressed mice

Figure 4 shows the effects of Neurotropin on platelet and megakaryocyte counts. Neuro- tropin, $100 \mathrm{mg} / \mathrm{kg} \times 1$ and 7 , had no significant influence on the counts for both cell types in non-stressed mice. In SART-stressed mice, the decreased platelet count and increased megakaryocyte count were uniformly and dose-dependently inhibited by consecutive preadministrations of Neurotropin at 50 and $100 \mathrm{mg} / \mathrm{kg}$, although a single dose of $100 \mathrm{mg} / \mathrm{kg}$ failed to cause any such effect. Daily treatment with $100 \mathrm{mg} / \mathrm{kg}$. in particular, remarkably prevented the changes in the counts for both cell types to normal levels.

As presented in Fig. 5, daily administration of alprazolam at 1 or $2 \mathrm{mg} / \mathrm{kg}$, doses found to have no influence in unstressed mice, tended

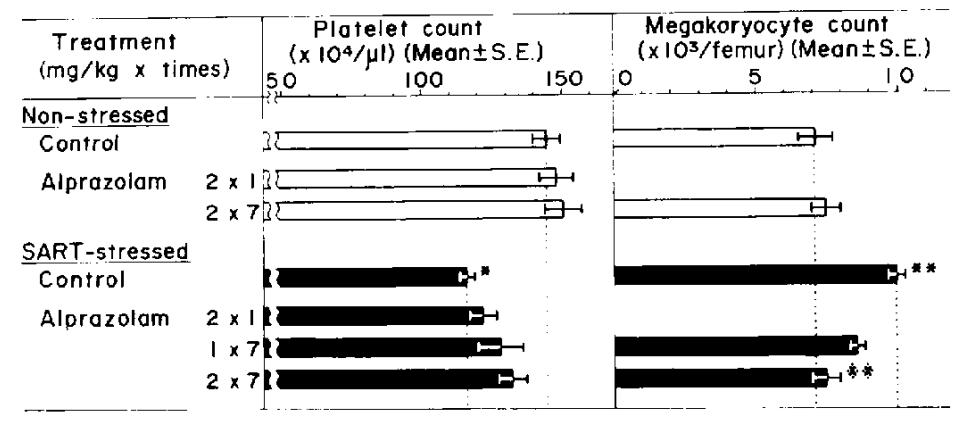

Fig. 5. Effect of alprazolam on SART stress-induced changes in platelet and megakaryocyte counts in mice. $n=5-8$. $P<0.05,{ }^{*} P<0.01$, compared to the non-stressed control, $* P<0.01$, compared to the SART-stressed control.

Table 1. Effect of drugs on SART stress-mduced prolongation of bleeding time

\begin{tabular}{|c|c|c|c|c|}
\hline \multicolumn{2}{|c|}{$\begin{array}{l}\text { Drug and dose } \\
\text { (mg/kg } x \text { times) }\end{array}$} & \multicolumn{2}{|c|}{ Bleeding time (sec) (Mean \pm S.E.) } & \multirow[t]{2}{*}{$\begin{array}{c}\text { Inhibition } \\
(\%)\end{array}$} \\
\hline Control & & $60.2 \pm 2.9$ & $130 . \overline{5 \pm 6.1^{* *}}$ & \\
\hline \multirow[t]{5}{*}{ Neurotropin } & $50 \times 1$ & & $106.6 \pm 10.0$ & 34.0 \\
\hline & $100 \times 1$ & $57.8 \pm 6.0$ & $94.7 \pm 8.1 \%$ & 51.0 \\
\hline & $200 \times 1$ & $63.4 \pm 6.0$ & $96.8 \pm 9.8$ & 47.9 \\
\hline & $50 \times 7$ & & $96.5 \pm 12.5$ & 48.4 \\
\hline & $1.00 \times 7$ & $53.7 \pm 10.5$ & $68.8=5.4 \%$ & 87.8 \\
\hline \multirow[t]{3}{*}{ Alprazolam } & $2 \times 1$ & $66.4 \pm 5.6$ & $115.0 \pm 12.8$ & 22.0 \\
\hline & $1 \times 7$ & & $110.6 \pm 11.8$ & 28.2 \\
\hline & $2 \times 7$ & $59.2 \pm 6.5$ & $85.0 \pm 9.9 \%$ & 64.8 \\
\hline
\end{tabular}

$\%$ Inhibition $=\frac{\text { (control value }- \text { drug-treated value) in SART-stressed groups }}{\text { difference between non- and SART-stressed control values }} \times 100$

Route: Neurotropin, i.p.; Alprazolam, p.o. No. of mice: $22 /$ control and 4-13/drug-treated group. $* P<0.01$, compared to the non-stressed control; $* \mathrm{P}<0.05, \% \mathrm{P}<0.01$, compared to the SART-stressed control. 
to inhibit the decrease in platelet count and significantly blocked the increase in megakaryocyte count in SART-stressed mice. As $2 \mathrm{mg} / \mathrm{kg}$ of alprazolam elicited significant behavioral suppression, doses greater than 2 $\mathrm{mg} / \mathrm{kg}$ were not given to mice.

Table 1 represents the effect of the two drugs on bleeding time. Consecutive administrations of either Neurotropin or alprazolam inhibited the SART stress-induced prolongation of bleeding time without any notable influence on non-stressed mice. Chronic treatment with Neurotropin, in particular. resulted in considerable inhibition to a level close to that in normal mice. In addition. single doses of Neurotropin, 50 and $100 \mathrm{mg} /$ $\mathrm{kg}$, shortened the bleeding time of SARTstressed mice in a dose-dependent manner. although a larger dose. $200 \mathrm{mg} / \mathrm{kg}$, failed to cause any additional effect.

\section{Discussion}

The data obtained from hematological comparisons in mice subjected to various forms of stress indicated that SART-stressed mice may be characterized by the presence of thrombocytopenia.

Numerous clinical investigations have shown that stress can cause quantitative and/ or qualitative alterations in human platelets ( 1 . $3,22-26$ ). For example, platelet count has been reported to increase immediately after exercise for short periods but found to show no change after prolonged exercise (22-24). There is also some evidence to suggest that platelet adhesiveness and/or aggregability may transiently increase following exercise and acute mental stress (1, 25, 26). In laboratory animals, however, studies concerning the relation between stress and platelets have been comparatively few, although some investigators have reported that some special forms of stress such as hypoxia (27). magnetic fields (28) and hypothermia (29) lead to thrombocytopenia.

In the present study, the direction of changes in platelet count differed according to the type of stress, and decreased platelet counts were recognized in mice exposed to SART stress and RWIS. The thrombocytopenia caused by RWIS may be essentially attributable to concomitant hypothermia. In addition, as RWIS-loaded animals show extensive severe abnormalities including strikingly decreased blood flow (8) and enhanced blood viscosity, such other factors should also be taken into consideration. A SART-stressed condition is produced by alteration of the environmental temperature, which may be encountered by humans in daily life, unlike exceptional forms of stress such as hypoxia, magnetic fields and RWIS, therefore supporting the possibility that thrombocytopenia is characteristic of SART stress and useful for making distinctions from other common stresses.

Thus, the relation between stress and platelets is complicated, and the effects of severity and duration of stress and species differences still remain to be elucidated.

The findings that $\mathrm{RBC}, \mathrm{Hb}, \mathrm{Ht}$ and $\mathrm{GB}$ were increased by any form of stress with the exception of cold exposure may have been due to hemoconcentration occurring in what has been called a stage of the alarm reaction in the "general adaptation syndrome" described by Selye (30). In any case, these phenomena are not peculiar to SART stress.

The circulating platelet count is essentially regulated by production in the bone marrow, destruction and accumulation in the spleen, and intravascular consumption.

Splenectomy failed to block the decrease in platelet count resulting from SART stress, thereby suggesting that thrombocytopenia is not a result of the enhancement of splenic functions including the destruction and accumulation of platelets.

The bone marrow megakaryocyte count is an important parameter reflecting platelet producibility and is practically utilized for clinical diagnosis. The augmented megakaryocyte count in SART-stressed mice is thought to indicate enhanced production of platelets, although other parameters such as cell size and ploidy should also be taken into consideration.

In conclusion, it appears that the thrombocytopenia caused by SART stress does not result from enhanced destruction or accumulation in the spleen or suppressed production in the bone marrow.

Furthermore, the above evidence suggests that platelet production in SART-stressed 
mice may be accelerated in order to com pensate for the decrease in circulating platelet count. In addition, such a possibility might lead to the speculation that SART stressinduced thrombocytopenia is not associated with abnormal distribution or storage of platelets. The resolution of the mechanism responsible for the thrombocytopenia will require more detailed studies on platelet consumption, especially intravascular aggregation, and other aspects.

Of interest is that the development of megakaryocytosis completely corresponded to that of thrombocytopenia during SART stress. If this megakaryocytosis was a compensatory event secondary to thrombocytopenia, however, the latter would precede the former. Another possibility is that differentiation of megakaryocytes may be suppressed. resulting in thrombocytopenia. For the present, the possibility cannot be excluded that the megakaryocytosis was independent of the thrombocytopenia, since the factor responsible for triggering the former is unknown.

The prolonged bleeding time observed in SART-stressed mice is compatible with the decreased platelet count. However, the extent of the change in bleeding time (over twice the normal level) seems too large, considering the change in platelet count (a $25 \%$ decrease). Further studies will be necessary to explain this inconsistency.

The finding that chronic treatment with either Neurotropin or alprazolam prevented the decrease in platelet count, increase in megakaryocyte count and prolonged bleeding time in SART-stressed mice supports previous studies in which both these drugs were effective in moderating SART stress-induced abnormal symptoms $(6-9,12,14,15)$.

Alprazolam has been reported to have an antagonistic effect against platelet-activating factor (PAF) (31). However, it is unlikely that such a mechanism would have been responsible for the inhibitory effect on thrombocytopenia observed in this study. because mouse platelets have been shown to be generally insensitive to PAF (32).

It is noteworthy that a single dose of Neurotropin shortened the bleeding time prolonged by SART stress. With respect to the effect of Neurotropin on hemostasis, there are a few reports. In one study, Neurotropin inhibited in vitro aggregation of rabbit platelets (33): another study indicated that Neurotropin induced in vivo shape changes in human platelets (34). In any case, our study suggests that Neurotropin may directly affect hemostatic systems including platelet aggregation. In addition, it is of special interest that acute treatment with Neurotropin, as with chronic treatment, produced an effect in SART-stressed mice without influencing unstressed mice.

\section{References}

1 Cash, J.D.: Platelets, fibrinolysis and stress. Thromb. Diath. Haemorrh. [Supp.] No. 51. p. 93-104 (1972)

2 Beattie, D.: Physiological changes in rats exposed to cold/restraint stress. Life Sci. 23 , 2307-2314 (1978)

3 Ohri, V.C., Chatterji, J.C., Das, B.K., Akhtar, M., Tewari, S.C., Bhattacharji, P. and Behl, A.: Effect of submaximal exercise on haematocrit. platelet count, platelet aggregation and blood fibrinogen levels. J. Sports Med. 23. 127-130 (1983)

4 Kita, T., Hata, T., Yoneda, R. and Okage, T.: Stress state caused by alternation of rhythm in environmental temperature, and the functional disorders in mice and rats. Folia Pharmacol. Japon. 71, 195-210 (1975) (Abs. in English)

5 Hata, T., Kita, T., Itoh, E. and Harada, N.: Experimental studies on optimal conditions of loading SART stress (repeated cold stress) upon animals. Japan. J. Psychosom. Med. 24. 257-266 (1984)

6 Kita. T.. Hata, T., Itoh, E. and Namimatsu, A. Testing methods for vegetative syndrome in the rat and effects of Neurotropin and other drugs. Japan. J. Psychosom. Med. 23, 61-68 (1983)

7 Hata, T., Kita, T., Namimatsu, A., Itoh, E. and Oda, Y.: Changes of blood pressure and regional blood flow in SART rats and drug actions on these changes. Folia Pharmacol. Japon. 79, 335-342 (1982) (Abs. in English)

8 Hata, T., Kita, T., Kawabata, A., Itoh, E. and Nishimura, Y.: Changes of tissue blood flow in mice loaded with SART (repeated cold) stress or restraint and water immersion stress and the effect of administered Neurotropin. Japan. J. Pharmacol. 41, 69-79 (1986)

9 Hata, T., Kita, T., Itoh, E. and Namimatsu, A.: Changes of the function in the heart of SART stressed (repeated cold stressed) mice and the 
action of Neurotropin on these changes. Folia Pharmacol. Japcn. 79, 487-492 (1982) (Abs, in English)

10 Hata, T., Nishimura, Y., Kita, T., Kawabata, A. and Itoh, E.: Electrocorticogram in rats loaded with SART stress (repeated cold stress). Japan. J. Pharmacol, 45, 365-372 (1987)

11 Kita, T., Hata, T., lida, J., Yoneda, R. and Ishida, S.: Decrease in pain threshold in SART stressed mice. Japan. J. Pharmacol. 29, 479-482 (1979)

12 Hata, T., Kita, T., Higashiguchi, T. and Ichida, S.: Total acetylcholine content, and activities of choline acetyltransferase and acetylchalinesterase in brain and duodenum of SART-stressed (repeated cold-stressed) rat. Japan. J. Pharmacol. 41, 475-485 (1986)

13 Hata, T., Kita, T., Kamanaka, Y., Honda, S., Kakehi, K., Kawabata, A. and Itoh, E.: Catecholamine levels in the brain of SART (repeated cold) -stressed rats. J. Auton. Pharmacol. 7, 257266 (1987)

14 Yoneda, R., Sugahara, K., Kita, T., Hata, T., lida, J., Ishida, S. and Ohba, Y.: Organ weights and hematological observation in SART stressed rats or mice, and effects of extracts isolated from vaccinia virus inoculated and inflamed skin of rabbits (Neurotropin ${ }^{2}$ ). Pharmacometrics 18 , 587-596 (1979) (Abs. in English)

15 Hata, T., Kita, T. and Nishimura, Y.: Effects of Neurotropin and other drugs on the EEG alterations in SART-stressed (repeated cold-stressed) rats. Folia Pharmacol. Japon. 91, 163-171 (1988) (Abs. in English)

16 Kita, T., Hata, T., Harada, N. and Itoh, E.: An abnormal ECG and the adrenaline-induced arrhythmias in restraint and water immersion stressed mice and effects of oxprenolol on them. Folia Pharmacol. Japon. 83, 373-382 (1984) (Abs. in English)

17 Komiya, E.: Clinical Hematology. p. 86-88. Nanzarido, Tokyo (1960) (in Japanese)

18 Brecher, G. and Cronkite, E.P.: Morphology and enumeration of human blood platelets. J. Appl. Physiol. 3, 365-385 (1950)

19 Cohen, M., Simmons, D.J. and Joist, J.H.: Diurnal hemostatic changes in the rat. Thromb. Res. 12, 965-971 (1978)

20 Nomura, T.: Differentiation of megakaryocytes. Acta Haematol. Japon. 37, 627-637 (1974) (Abs. in English)

21 O'Neil, C.: Examination of the causes of early pregnancy-associated thrombocytopenia in mice. J. Reprod. Fertil. 73, 567-577 (1985)

22 Sarajas, H.S.S., Konttinen, A. and Frick, M.H.: Thrombocytosis evoked by exercise. Nature 192, $721-722$ (1961)

23 Pegrum, G.D., Harrison, K.M., Shaw, S., Haselton, A. and Wolff, S.: Effect of prolonged exercise on platelet adhesiveness. Nature 213, 301-302 (1967)

24 Bennet, P.N.: Effect of physical exercise on platelet adhesiveness. Scand. J. Haematol. 9, 138-141 (1972)

25 Haft, J.l.: Catecholamine, stress, platelets and coronary artery disease. in Platelets. Prostaglandins and Cardiovascular Disease, Edited by Mehta, J. and Mehta, P.F., p. 265-277, Futura, New York (1981)

26 Colwell, J.A.: Effects of exercise on platelet function, coagulation, and fibrinolysis. Diabetes Metab. Rev. 1, 501-512 (1986)

27 Jackson, C.W. and Edwards, C.C.: Biphasic thrombopoietic response to severe hypobaric hypoxia. Br. J. Haematol. 35, 233-244 (1977)

28 Gorczynska. E. and Wegrzynowicz, R.: The effect of magnetic fields on platelets, blood coagulation and fibrinolysis in guinea pigs. Physiol. Chem. Phys. Med. NMR 15, 459-468 (1983)

29 Yoshihara, H., Yamamoto, T. and Mihara, H.: Changes in coagulation and fibrinolysis occurring in dogs during hypothermia. Thromb. Res, 37, 503-512(1985)

30 Selye, H: A syndrome produced by diverse nocuous agents. Nature 138, 32 (1936)

31 Kornecki, E. Ehrlich, Y.H. and Lenox, R.H.: Platelet-activating factor-induced aggregation of human platelets specifically inhibited by triazolobenzodiazepines. Science 226, 1454-1456 (1984)

32 Namm, D.H., Tadepalli, A.S. and High, J.A.: Species specificity of the platelet response to 1 -o-alkyl-2 - acetyl-sn-glycero-3-phosphocholine. Thromb. Res. 25, 341-350 (1982)

33 Nakamura, M., Kosugi, T. and Kinjo, K.: The effect of Neurotropin upon the aggregation of rabbit platelets. Medicine and Biology 108, 315318 (1984) (in Japanese)

34 Arimori, S., Matsumura, Y. and Takeuchi, S.: The effect of intravenous infusion of Neurotropin ${ }^{*}$ on human platelets. Medicine and Biology 102, 9-11 (1981) (in Japanese) 